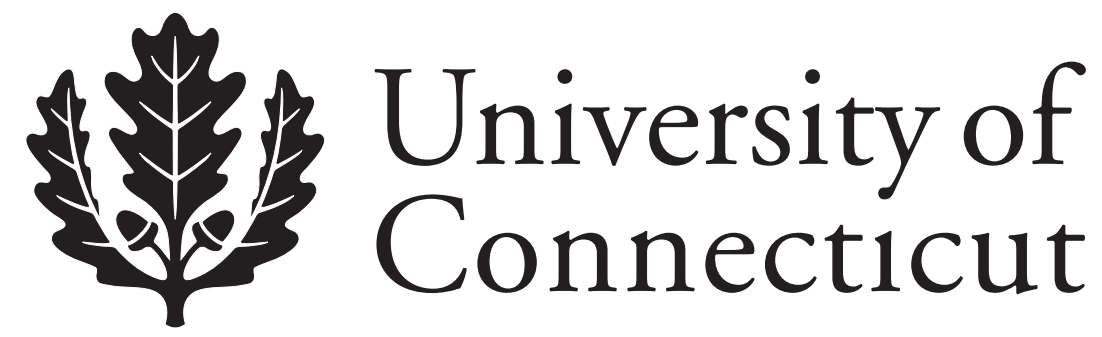

Department of Economics Working Paper Series

\title{
Online Graphing Activity for Principles of Economics Courses
}

Oskar R. Harmon

University of Connecticut

James Lambrinos

Union Graduate College

Working Paper 2010-31

December 2010

341 Mansfield Road, Unit 1063

Storrs, CT 06269-1063

Phone: (860) 486-3022

Fax: (860) 486-4463

http://www.econ.uconn.edu/

This working paper is indexed on RePEc, http://repec.org/ 


\begin{abstract}
This paper describes how an online drawing program and bulletin board are used to create active learning activities for a principles of economics class. In the activity the student downloads an initial diagram that sets up a textbook principles scenario. The student uses an image-editing program to complete the diagram, so that it represents the outcome predicted in the textbook and posts it to a bulletin board. The tools for the activity: SumoPaint.com, and WikiSpaces.com; are free and available on the Internet. By appropriately adjusting the information included in the initial diagram, there is flexibility to adjust the difficulty level, for high school AP courses or college level courses, and to adapt the exercise to a range of different classroom formats and varied physical locations of the student.
\end{abstract}

Journal of Economic Literature Classification: A2

Keywords: Online, Blended, Hybrid, Teaching Economics 


\section{Online Graphing Activity for Principles of Economics Courses}

This article describes how an online drawing program and an online bulletin board can be used to create active learning activities for a principles of economics class. In the activity the student copies an initial diagram that sets up a standard textbook principles scenario. To finish the activity the student uses an image editing program to complete the diagram, so that it represents the outcome predicted in the textbook analysis, and posts it to an online bulletin board. The tools for the activity: SumoPaint.com, and WikiSpaces.com; are available on the internet and are free to use. It is the of $21^{\text {st }}$ century equivalent classroom chalk talk.

Exhibit 1 shows a representative example of the activities we designed using these tools. In the sample activity the student is given a diagram with the usual short run cost curves of marginal cost, average total cost, and average variable cost, in two panels. The activity is to draw in demand and marginal revenue curves to represent the short equilibrium of positive economic profits for a perfectly firm (left-side graph) and a monopoly firm (rightside graph). The student is directed to copy the image to the desktop, and open the image in SumoPaint.com.

\section{Exhibit 1}

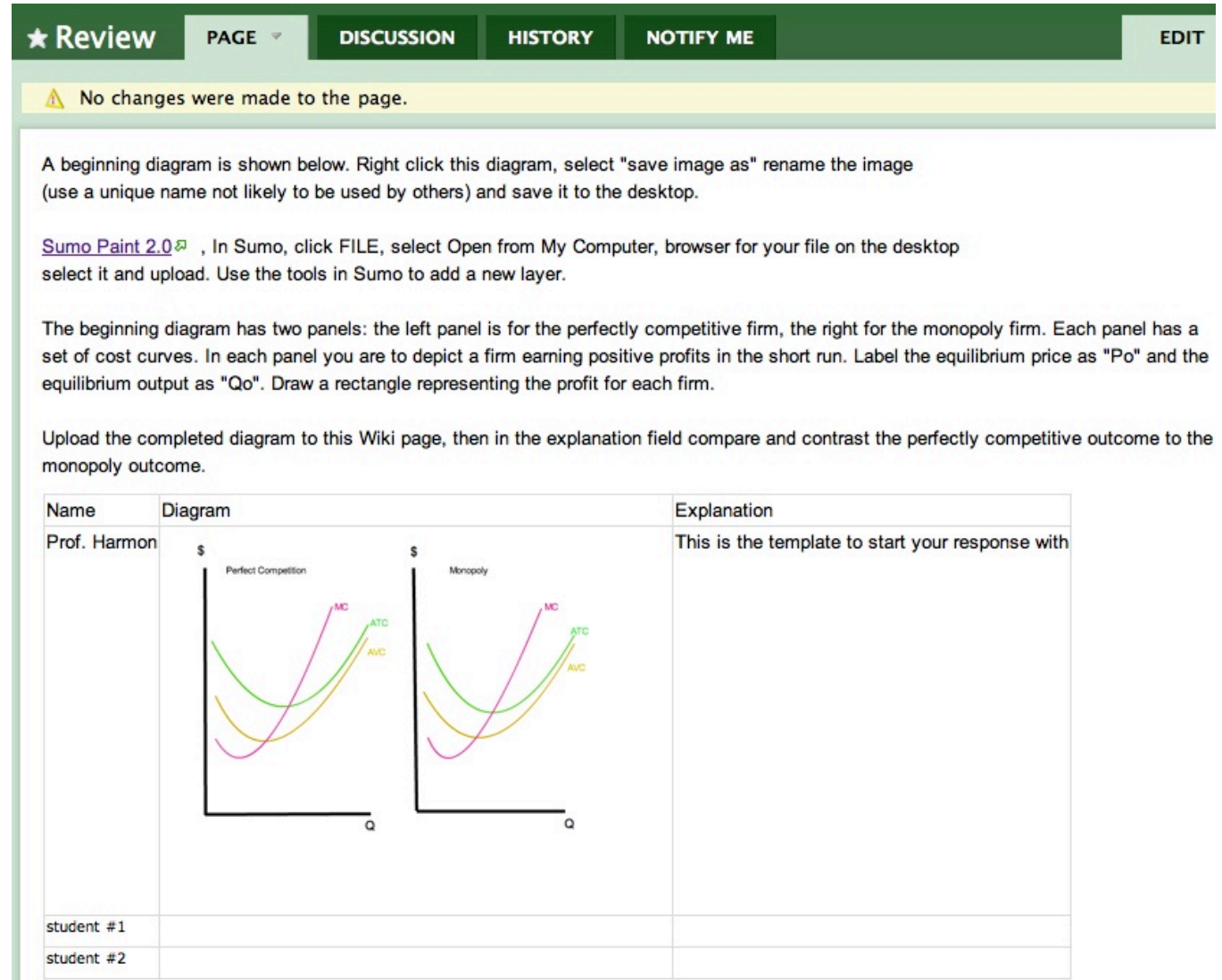

Using the 
drawing tools in SumoPaint.com the student draws in the appropriate demand and marginal revenue cures, labels the equilibrium price and quantities and then uploads the image to a bulletin board in WikiSpaces.com. In the bulletin board (Exhibit 1) there is cell opposite the student's name to post the diagram and next to it a cell to post the explanation of the diagram.

There is flexibility to adjust the difficulty level of this exercise. For example, it can be increased by providing in the base diagram only the axis grid without the cost curves, or decreased by providing the demand curves, the marginal revenue curves, and the cost curves. There is flexibility to adjust the level of student interaction. Because the bulletin board can be viewed and edited by students in the class, students can post individually or as members of a group, can learn from each other's posts, and they can post constructive comments. The user ID need not be identified in the bulletin board, posts can be made anonymously, or using favorite screen names or avatars.

There is flexibility to adapt the exercise to a range of different classroom formats and varied physical locations of the student's. For example, it can be used in an online class where students have broadband access and a desk surface to comfortably work on their computer. In this setting the activity can have a relatively high degree of difficulty and high student interaction. Or, at the other end of the spectrum, it can be used in an face-to-face class where students, using mini laptop or a smart phone that runs flash, are seated with limited desktop surfaces, and the room has equipment to project the instructor computer display on an overhead screen. In this setting the activity will have minimal typing and drawing demands, and the instructor can discuss with the class the graphs as they are posted.

The remainder of the article is a quick start guide on how to use the tools SumoPaint.com and WikiSpaces.com to design these type of exercises. We tried several other free drawing programs and public discussion boards, the ones used here we found to be the easiest to use. You may well find similar tools that work better for your situation. For SumoPaint.com requires Flash software and iPhones and iPads currently don't run. However, the approach of combining image editing, and posting to an online bulletin board is an innovation we haven't seen elsewhere. It is our hope you will find this approach as useful and engaging for students as we have found for our classes.

\section{Quick Start to SumoPaint.com}

This is a free online tool. The url www.sumopaint.com/app opens up to a page similar to that shown in Exhibit 2. (Exhibit 2 displays the answer diagram for this activity, at the startup of SumoPaint only a white drawing space is displayed.) The column on the left is the palette of drawing tools. The row at the top of Exhibit 2 displays image control

commands (File: File etc.) and the second row displays controls for the properties of the drawing tools, which change depending on the drawing tool selected. In the right hand column are pallets for color and image layers. 


\section{Exhibit 2}

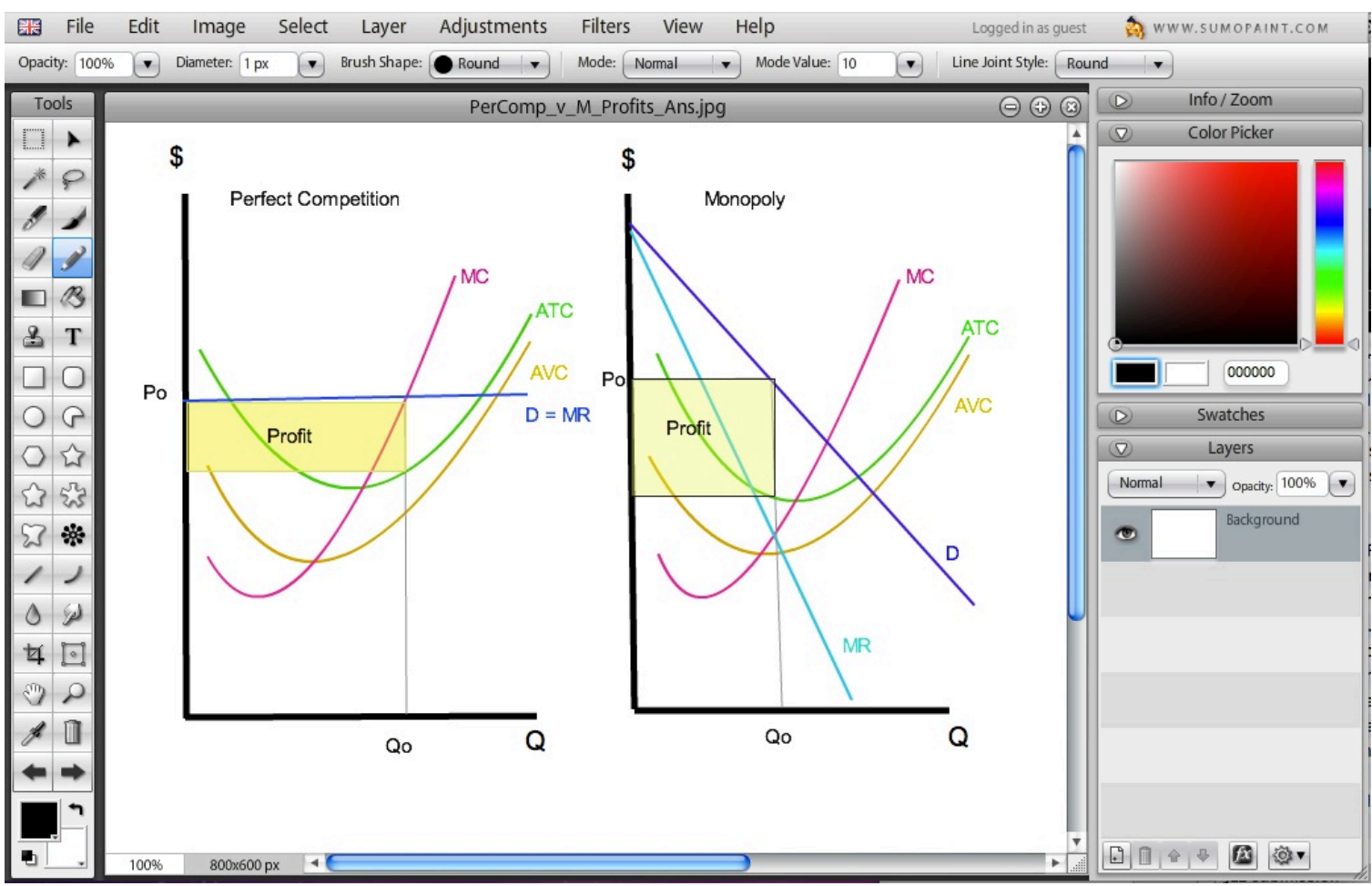

The drawing tools that we have found most useful this activity are: Eraser, Pencil, Line, Curved Line, Text, Rectangle, Undo/Redo. A mouse-over the tool pallet icon, displays the tool name.

When the Eraser tool is selected the properties of the tool are shown in the tool properties bar in the top second row. The tool can be adjusted for more precision by increasing the

Flow property and decreasing the Diameter property: Flow: $33 \% \quad$ Diameter: $30 \mathrm{px} \quad(30$ px is good for erasing a big area). As with all the numeric properties the setting is adjusted by a sliding bar or simply typing in the desired number. The Undo and the Redo tools, which are the icons with arrows pointing left/right: $|\overrightarrow{\mid}|$; help edit out mistakes.

Three tools can be used to draw straight and curved lines: the Pencil tool, the Line tool, and the Curve tool. For these tools the thickness of the line can be set by typing a number in the Diameter box: Opacity: $100 \%$ Diameter: $1 \mathrm{px}$ clicking in the Color Picker pallet in the right side column. The selected color is displayed at the bottom of the tool pallet. The opacity property is a quick way to change a black line to grey. The other properties don't visibly change the display of small diameter lines.

The Text tool can be used for labels. The size and color are set in the properties bar.

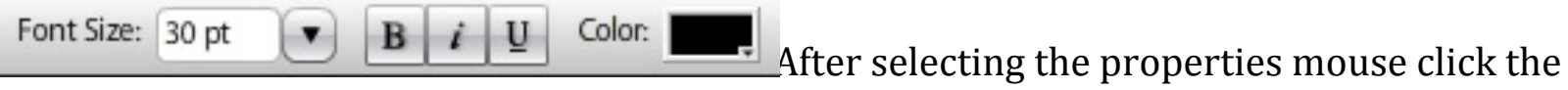


location desired for the text. A text rectangle appears, click inside that to enter text. Then click the Text tool icon to complete the creation of the label.

For the Rectangle tool click the down arrow next to the Fill property:

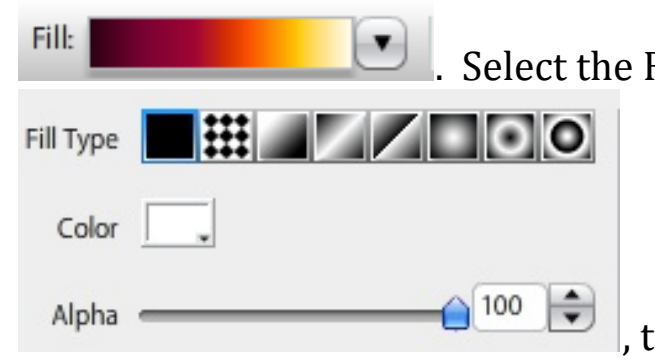

below the black Fill Type rectangle. Decreasing the Alpha property (directly below Color) lets lines below the rectangle show through. There is no Triangle tool, but use of the Rectangle in combination with the Eraser tool can create a triangle shape.

The Layers function, located at the bottom of the right side column can keep parts of the diagram separate from others as the diagram is drawn. To add a layer click the icon that resembles a page: 5 (a mouse-over will display the name) and is located at the very bottom of the right side column. From experience, drawing a diagram in layers makes it easier to make corrections, and to create the initial and final images.

To save the image click the File command in the top row of image controls above the image editing space. For the option Save to My Computer there are three format choices: SUMO, PNG, and JPG. The SUMO format can be opened in the sumopaint software and retains control over the layers, the JPG format flattens the layers. Once the "answer" diagram is created, deselect the visibility of the layers (click the eyeball: $c$ that is opposite the layer which is shown in the Layers box in the right side column) you do not want the students to see (i.e. the part of the diagram you want them to draw), and make a screen capture of the visible diagram. (To make a screen capture on a Mac use the key strokes:

Command/Shift/4; on a PC use the key strokes Ctrl/Alt/PrintScreen to capture the image, open the Paint Program, click Paste, then use the Crop command to capture just the diagram). This image is what is uploaded to WikiSPaces.com as the initial image for the students to work with.

\section{Quick Start to WikiSPaces.com}

This also is a free online tool. Go to wikispaces.com, scroll down to the bottom of the page, select: Wikis for Individuals and Groups. Complete the fields for Username, Password, and E-mail. Click: Join Now, and you now have a free Wikispaces account!

\section{Exhibit 3}


Controls for the wikispaces account are in the top frame of the wiki page. (Exhibit 3). Within the wikispaces account (My Account: has the settings for password etc.) the user can have several wikis (My Wikis: lists the ones created), and each wiki can have several pages. A wiki is created by clicking the button: Create a New Wiki. It takes you to a page where you name the wiki and set the access to public or private. The name format is letters and hyphens, for example: Principles-of-Economics.wikispaces.com. The newly created wiki appears in the drop down list of "My Wikis", like this:

Principles-of-Economics

My Wikis

Click the name Principles-of Economics brings up a Welcome page.

\section{Exhibit 4}

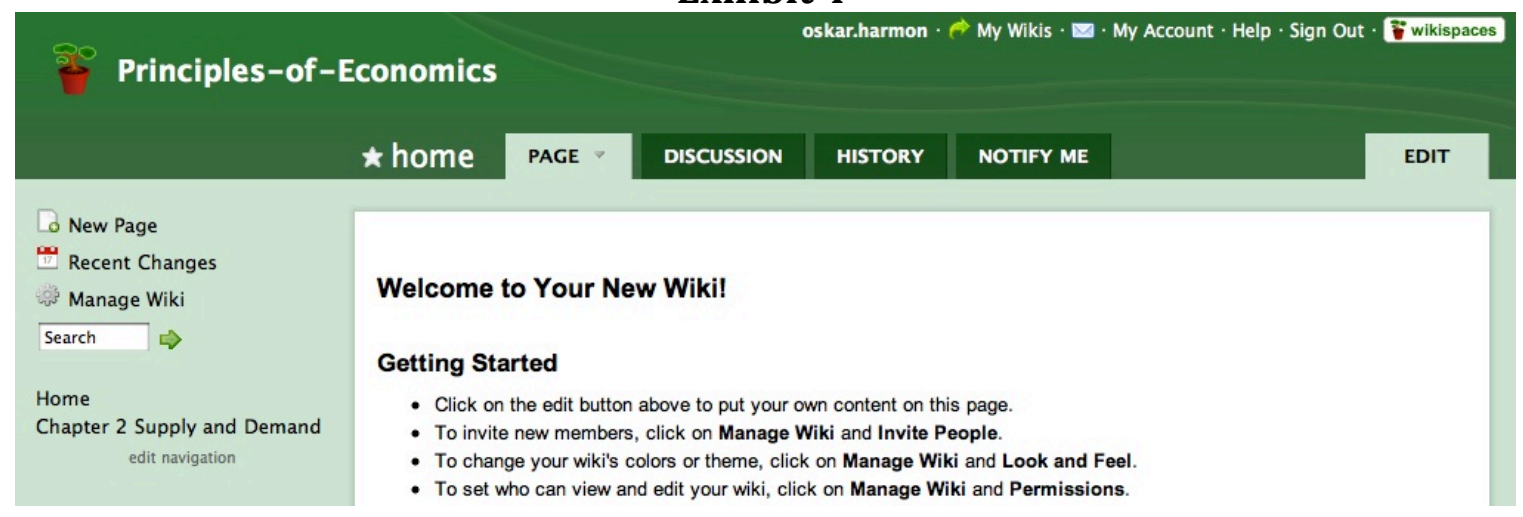

The welcome page is displayed in the middle frame, and is linked from the left side column with the title "Home" show below the "search" box. Additional pages are created by clicking the icon for New Page: 0 New Page. Enter a page name:

Page Name Chapter 2 Supply and Demand

under the first link for "Home".

and the link will appear in the left side frame

Data is entered on the page by a click on the Edit icon: EDIT . In the edit mode, shown in Exhibit 5,

Exhibit 5

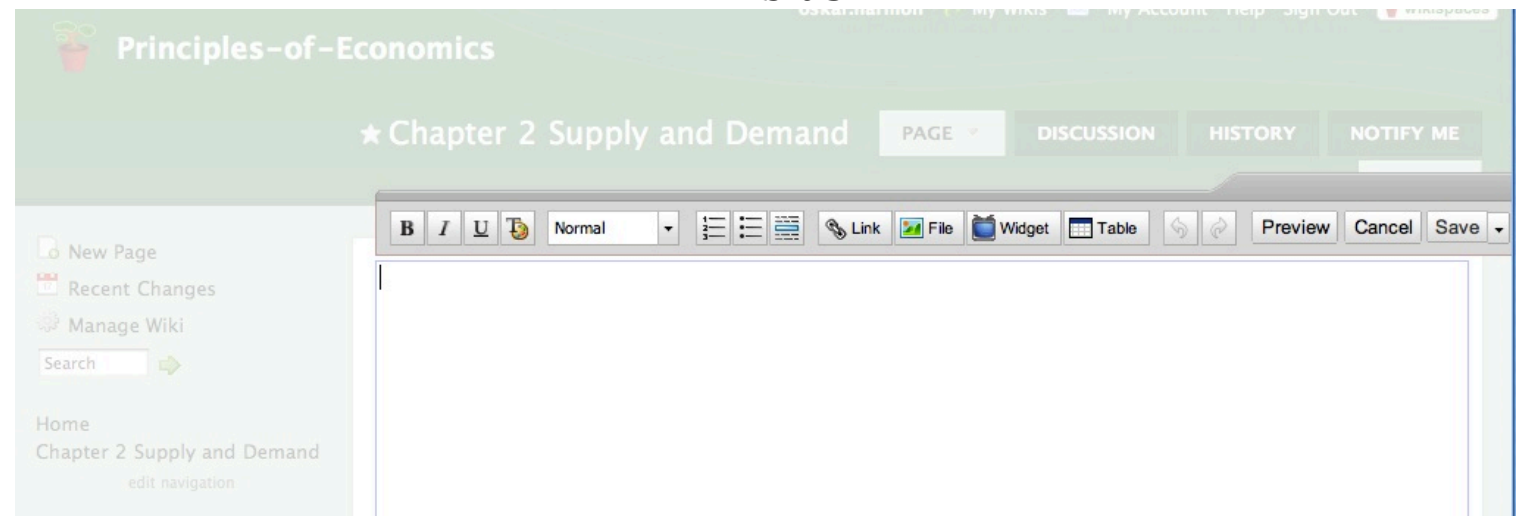


instructions for the exercise can be entered. Using the Table control: $\square$ Table and the File control: File a page similar to that in Exhibit 1 can be created. (Initially enter some typing before closing the page, otherwise the page does not get created.) The upload process for the student is the same as the instructor: click Edit, click a cell on the grid, click File, then browse for the image on the hard drive.

The invitations to join the wiki, as well as other properties are set in the control Manage Wiki, $w^{3}$ Manage Wiki see Exhibit 6.

\section{Exhibit 6}

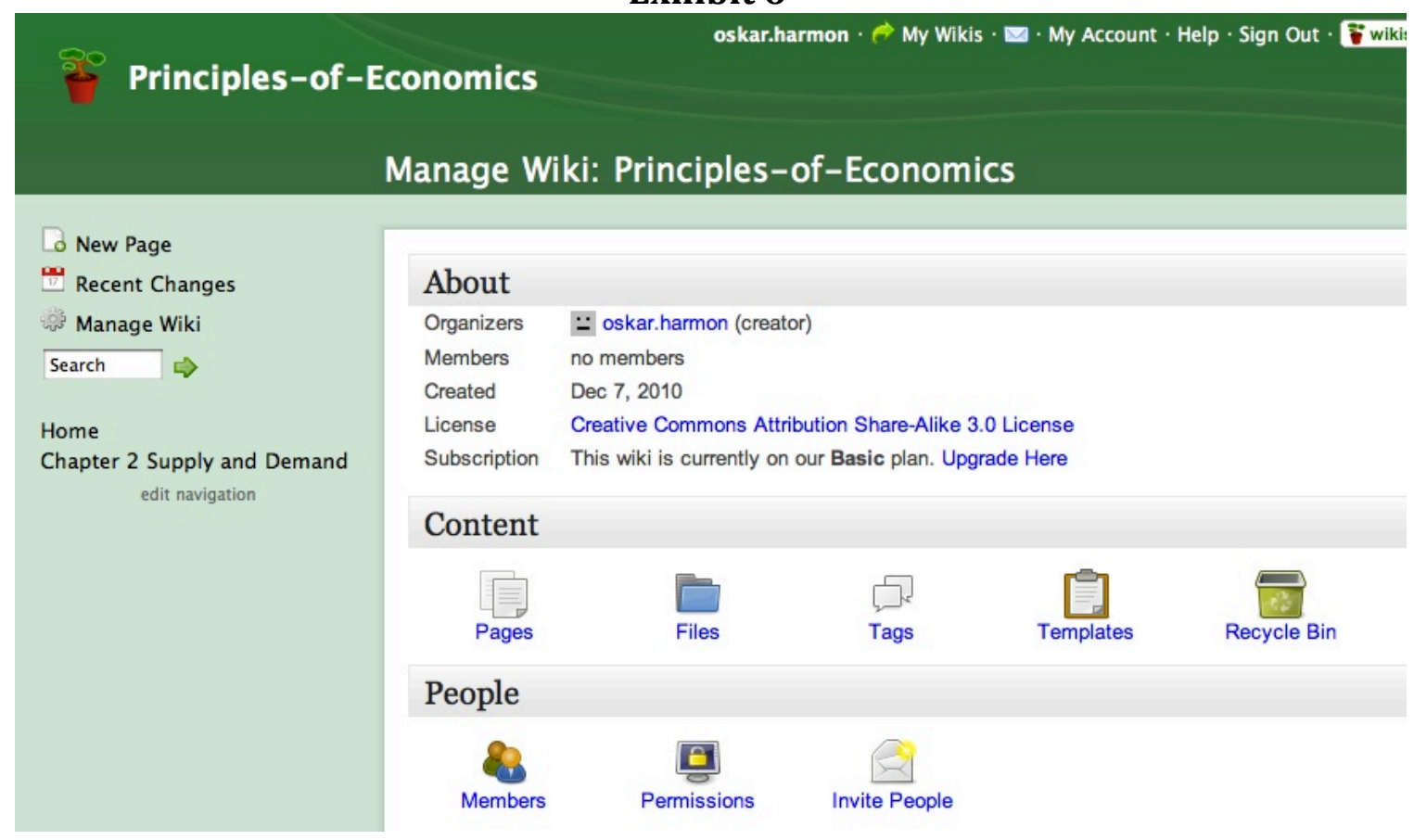

To send invitations for students to join Click the icon Invite People: Invite People; a dialogue box

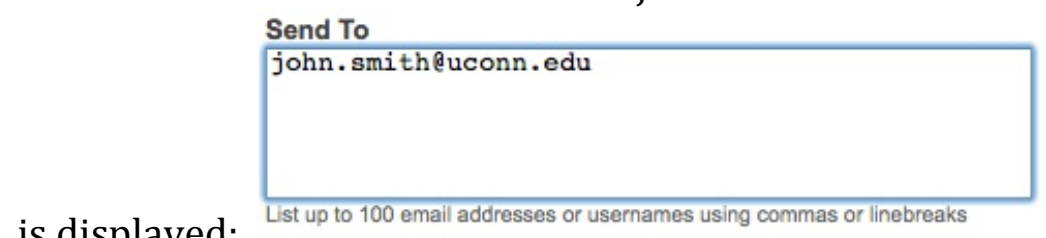

is displayed:

, then enter the email addresses.

In the free account, the board is "public" to anyone, but only students within the class can edit the pages. Private boards are available at a cost. Students upload images by same process as instructor. The process is straight forward and the only problems we have had are operator errors of: students can make the mistake of posting in the wrong cell, posting over another student's post, or using the same image name and overwriting another's image post. Exhibit 7 below has a set of instructions to the student for joining and using the Wiki. 


\section{Exhibit 7}

\section{How to Join Our Class Wiki}

Step 1: Go to www.econ1201-winter-2011.wikispaces.com/. (Note U cn view the class Wiki, but no EDIT it yet, that is what you accomplish in the below steps.)

Step 2: In the left navigation frame click "Join this Wiki".

Step 3: A new page is displayed "Welcome to Wikispaces". Sign in if you already have an account and go to Step 7, otherwise go to Step 4

Step 4: Click "Make a new account". For the user name use the name you registered in the with, for the password use your favorite one, for the email address use your university email address, then click "Join".

Step 5: An email is immediately sent to your Uconn email account, in that email click the confirmation link.

Step 6: Go back to www.econ1201-winter-2011.wikispaces.com/,

Step 7: In the left navigation frame click on "Join this Wiki", and sign in. On the following page in the Comment (it says optional but it is not optional) field enter your username, your class (Econ 1201), your affiliation (UConn), then click "Request Membership".

Step 8: An email is sent to the instructor (Prof. Harmon) to accept your account. Check your Uconn email account account periodically to receive confirmation. If the join request is not accepted, most likely you entered a user name the instructor doesn't recognize, so repeat Step 7, or email the instructor at: harmon@uconn.edu.

Step 9: Go back to www.econ1201-winter-2011.wikispaces.com/,, and follow the Getting Started instructions in the center frame.

\section{Getting Started}

- The navigation frame on the left has pages corresponding to each session of the course

- Click the link for the session you want to visit

- After reading the into for that page, view the first entry

- When you are ready, click on the "EDIT"** button above and to right to put your own content on this page, in the table row with your name.

- Since we can all write to the page, be careful not to delete the content posted by others.

- Name your files with your name as a prefix, a hyphen, and a number, starting with 1. (No spaces are allowed in the file name.)

- Each wiki page has a different exercise

\section{Need Help?}

- Click on the help link above to learn more about how to use your wiki.

- If you come across some other useful help resources, please let me know. I've used this Wiki previously, but I still have a lot to explore in the tool! 


\section{RESOURCES:}

A useful web resource for SumoPaint is:

http://www.sumopaint.com/help/

A YouTube Video on a WikiSPaces Tutorial

http://www.youtube.com/watch?v=df2rC2QfvFc

A web site with resources for WikiSpaces:

http://educationalsoftware.wikispaces.com/Wikispaces+Tutorial

An excellent example Wiki:

http://teachartwiki.wikispaces.com/ 